\title{
Article
}

\section{Searching for two categories of target in dynamic visual displays impairs monitoring ability}

Muhl-Richardson, Alexander, Cornes, Katherine, Godwin, Hayward, Garner, Matthew, Hadwin, Julie, Liversedge, Simon Paul and Donnelly, Nicholas

Available at http://clok.uclan.ac.uk/22518/

Muhl-Richardson, Alexander, Cornes, Katherine, Godwin, Hayward, Garner, Matthew, Hadwin, Julie, Liversedge, Simon Paul ORCID: 0000-0002-8579-8546 and Donnelly, Nicholas (2018) Searching for two categories of target in dynamic visual displays impairs monitoring ability. Applied Cognitive Psychology . ISSN 0888-4080

It is advisable to refer to the publisher's version if you intend to cite from the work. http://dx.doi.org/10.1002/acp.3416

For more information about UCLan's research in this area go to

http://www.uclan.ac.uk/researchgroups/ and search for <name of research Group>.

For information about Research generally at UCLan please go to http://www.uclan.ac.uk/research/

All outputs in CLoK are protected by Intellectual Property Rights law, including Copyright law. Copyright, IPR and Moral Rights for the works on this site are retained by the individual authors and/or other copyright owners. Terms and conditions for use of this material are defined in the policies page. 
Searching for Two Categories of Target in Dynamic Visual Displays Impairs Monitoring Ability

Alex Muhl-Richardson ${ }^{1}$, Katherine Cornes ${ }^{2}$, Hayward J. Godwin ${ }^{1}$, Matthew Garner ${ }^{1}$, Julie A. Hadwin ${ }^{1}$, Simon P. Liversedge ${ }^{1}$ and Nick Donnelly ${ }^{1}$

${ }^{1}$ University of Southampton

${ }^{2}$ Defence Science and Technology Laboratory

Author Note

Alex Muhl-Richardson is now at the Division of Psychology, Nottingham Trent University, Nottingham, UK.

Correspondence should be addressed to Alex Muhl-Richardson, Division of Psychology, Nottingham Trent University, Nottingham, UK. Email: alexander.muhlrichardson@ntu.ac.uk. 


\begin{abstract}
Target onsets in dynamically changing displays can be predicted when contingencies exist between different stimulus states over time. In the present study, we examined predictive monitoring when participants searched dynamically changing displays of numbers and colored squares for a color target, a number target or both. Stimuli were presented in both contiguous and discrete spatial configurations. Response time (RT) and accuracy were recorded and evidence of predictive monitoring was assessed via first fixations and refixations of target-predictive stimuli. RTs to target onsets and evidence of predictive monitoring were reduced in dual-target, relative to single-target, conditions. Further, predictive monitoring did not speed RTs but was influenced by display configuration. In particular, discrete displays impaired monitoring for number targets in the dual-target condition. Implications exist for real-world visual tasks involving multiple target categories and for visual display design.
\end{abstract}

Acknowledgements: This work was supported by funding from the Defence Science and Technology Laboratory, UK via the National PhD Scheme.

Keywords: visual search, eye movements, dynamic search, dual-task, dual-target 
In real-world scenarios, searching and monitoring for targets often takes place in the context of information presented on electronic visual displays. In these situations, where information can change dynamically over time, effective target detection requires sustained monitoring. When the changes that occur over time are systematically related (e.g. incremental increases on a temperature scale as an area gets warmer), target detection may be aided by prioritizing the monitoring of those items that might soon become targets. We have previously discussed elsewhere (Donnelly et al., 2006) the example of geological imaging. In this domain, a geologist might be tasked with inspecting image slices of a 3D rock volume with the aim of identifying shale deposits, indicated by red, amongst sandstone and limestone, indicated by blue and white respectively. Variations in density are such that progression through successive image slices can involve gradual changes in hue and luminance. The presence of pink in one slice may therefore be an indicator of a forthcoming red target in a subsequent slice.

The extent to which specific items in dynamically changing displays support predictive monitoring has been explored in a recent study (Muhl-Richardson et al., in press). In this study, participants’ eye movements were recorded while they monitored displays of changing colored squares (that resembled heat-maps) for the onset of a specific color target. Perhaps unsurprisingly, participants did prioritize the monitoring of items that had a high probability of soon becoming targets, but only when color changes occurred according to a psychologically ordered color space. This predictive monitoring was characterized by both fixations and refixations of target-similar items yet to become a target.

One might expect predictive monitoring to speed target detection and increase target hit rate, however, Muhl-Richardson et al. found no evidence of these benefits in their task. In part, this is because predictive monitoring was associated with two types of error: (1) false alarms arising from premature responses to forthcoming targets and (2) misses after fixating a 
forthcoming target but not returning to it post-onset. This prior study showed how participants prioritized likely target locations for monitoring even when such monitoring did not always lead to a beneficial outcome.

The present study extends the work of Muhl-Richardson et al. (in press) to examine how predictive monitoring is influenced by the need to simultaneously monitor for two types of target drawn from different categories. Previous studies of dual-target search, where targets were defined within a single category, have found a 'dual-target cost' to response time, accuracy and search guidance (Menneer, Phillips, Donnelly, Barrett, \& Cave, 2004; Stroud, Menneer, Cave, \& Donnelly, 2012; Stroud, Menneer, Cave, Donnelly, \& Rayner, 2011).

Simultaneously searching for two categories of target can be thought of as a special case of task switching. While the overall task remains one of visual search and monitoring, the search system must be reconfigured in order to detect targets from different categories. Switching between two tasks incurs performance costs that arise from residual activation of previously active task sets, and the need to reconfigure target templates and stimulusresponse mappings (Meiran, 2000; Meiran, Chorev, \& Sapir, 2000; Pashler, 2000). These costs can sometimes be reduced, but not eliminated, if there is time to prepare for a switch and if there is no overlap in stimulus-response mappings between tasks (Kiesel et al., 2010; Ravizza \& Carter, 2008).

Detecting the onset of targets drawn from two categories is very likely to take longer and be less accurate relative to single category baselines. However, the question we explore in the present study is different: are the eye-movement markers of predictive monitoring, as defined by Muhl-Richardson et al (in press), found when participants must search and 
monitor for targets in two categories? To address this, we used a dynamic search and monitoring task involving displays of single-digit numbers and colored squares.

The study is motivated by real-world visual monitoring tasks that involve complex displays of multiple information sources across different categories. An example of this is viewing and decision-making when monitoring marine radar. Displays in marine radar must be carefully designed to present maps of varying scale, in addition to alphanumeric indicators of position, direction and speed. In the present study, we explore how spatial constraints may influence monitoring for specific numbers and colored squares. A simple manipulation of the spatial constraints of displays is to position stimulus sets so that they appear in a single contiguous configuration or in spatially discrete (i.e. separate) locations.

In the contiguous displays used in the present study, the number set was embedded within the color set. This configuration placed the center of each stimulus set in the same location, such that fixed monitoring from this region would place the number set in foveal vision and the color set further into the periphery. The contiguous configuration was contrasted with a spatially discrete display configuration where the color and number sets were located on opposing sides of the display (see Figure 1a). The two spatial configurations contrasted in many display parameters (e.g. clutter, spatial extent, necessity for shifts of spatial attention, the implicit prioritization given to one stimulus set over another). We reason that spatial configuration may have an important influence on monitoring for target onsets. For example, contiguous displays may enable monitoring with relatively few large overt shifts of attention, allowing more items to be monitored in parallel. However, contiguous displays might also be perceived as more cluttered than discrete configurations and impair monitoring (Lavie, 2006; Lavie, Beck, \& Konstantinou, 2014). 
We acknowledge the differences between the stimulus sets in terms of visual size, set size and the number of potential distractor identities. The properties of the number set (i.e. their overall size and number of items) were defined to fit within a contiguous configuration with the color set, while maintaining discriminability and we do not treat the color and number searches equally. From an applied perspective, this is representative of many visual scenarios where one stimulus set provides richer and more complex information than another, for example, the view of an external environment through a head mounted display. Different responses were also employed for each stimulus category in an attempt to minimize responsebased interference in dual-target conditions.

In the present study, dual-target search and single-target search for each of the two target categories were examined in each display configuration. The detection of target onsets was measured in terms of behavioral measures of hit rate, response time (RT) and false alarm (FA) rate. Eye movements were used to assess the ability to prioritize and predictively monitor potential targets, this was accomplished by examining first fixations to targets and forthcoming targets (as a function of the number of color or number steps from a target state), refixations of these stimuli, and the duration of visits to each stimulus array.

Based on previous studies of dual-target visual search, it was predicted that target detection would be worse (reduced hit rate and elevated RTs), and predictive first fixations and refixations reduced, in dual-target conditions (monitoring for color and number targets) relative to single-target conditions (monitoring for only color or number targets). Where predictive monitoring was possible, it was expected that there would be evidence of false alarms (arising from premature responses) and that misses might also be present. With regard to the effects of display configuration, it was anticipated that discrete displays would facilitate single-target searches due to the reduced likelihood of distraction. In contrast, we 
hypothesized that contiguous displays would facilitate dual-target searches due to the reduced need for large, overt, shifts of attention.

\section{Method}

\section{Participants}

26 participants (age range $=19$ to 42 years, $M=25.0$ years, $S D=5.4,14$ female) took part in the study. All were students at the University of Southampton and participated for partial fulfillment of a course requirement or were compensated with $£ 12$. Participants had normal visual acuity ( $>=1.0$ decimal at $70 \mathrm{~cm}$ ), tested using the Freiburg Visual Acuity Test (FrACT; Bach, 1996), and normal color vision, tested using the City University Color Vision Test $3^{\text {rd }}$ Edition (Fletcher, 1998).

\section{Apparatus}

Stimuli were displayed on a 21” CRT with a resolution of 1,024 x 768 and a refresh rate of $120 \mathrm{~Hz}$. This was controlled using a computer connected to an SR Research Eyelink 1000 monocular eye-tracker, operating at a sampling rate of 1,000 Hz. A nine-point calibration was used and was accepted only when none of the points had an error of more than $0.5^{\circ}$ of visual angle. Participants were seated $70 \mathrm{~cm}$ from the display in a chin rest and viewed the display binocularly, although only the right eye was tracked. The experiment was programmed using SR Research Experiment Builder with additional code in Python.

\section{Stimuli}

Basic properties. All displays consisted of color and number stimulus sets. In contiguous configurations, four digits were located within a rectangular set of 20 colored squares. This was centered at a point $7.29^{\circ}$ left or right of the display center. In discrete configurations, the same number and square sets were similarly centered on opposing sides. Squares, each $0.57^{\circ} \times 0.57^{\circ}$, were in irregular $6 \times 4$ sets with the central four stimuli removed. 
Squares never abutted and each appeared randomly offset within an area of $2.15^{\circ}$ x $2.15^{\circ}$. The maximum size of the color set was $11.61^{\circ} \mathrm{x} 7.35^{\circ}$. Number stimuli were displayed in a regular 2 x 2 set, with each digit no more than $1.08^{\circ} \times 1.14^{\circ}$. The maximum size of the number set was $2.79^{\circ} \times 3.77^{\circ}$. All of the above angles are visual angles calculated from the center of the display.

To simplify target assignment and because previous work did not suggest any systematic differences in search for different target colors (Muhl-Richardson et al., in press), two colors and all numbers were used as possible targets. A single number and a single color were pseudo-randomly assigned to each participant as targets, e.g. '8' and 'yellow', and this assignment remained constant across all applicable conditions. Stimuli underwent changes such that they systematically approached a target state, allowing the distractors to be used as predictive cues to the onset of targets. When distractors were within two steps of a target, for both colors and numbers, they potentially predicted a forthcoming target onset and were considered to be target-predictive distractors (TPDs). Participants were not explicitly informed of these contingencies (see also Muhl-Richardson et al., in press). Examination of whether targets were first fixated as TPDs forms the basis of later analysis of predictive monitoring. Within the color set, there were four potential locations for targets or TPDs. These were varied trial-by-trial, were spatially distinct and never included the four stimuli above or below the center. One, two or none of these four stimuli would become a target and the remainder would never reach the target color but, at the closest, a state one step from the target color. Within the number array, any three out of the four locations could become a target or TPD. Again, one, two or none of these would become a target and the remainder would never reach the target number but only a state one step from the target number. In all cases, targets and TPDs were reset to a distractor state if a response was made or after a varied delay (see below) and only one of each target type could be active at any time. All 
stimuli changed in single sequential steps through 16 colors or 10 digits. The color stimuli were all approximately equally spaced in color space and, with the exception of looping between ' 0 ' and ' 9 ', the numbers were equally semantically spaced (i.e. on a number line; see Figure 1 for further details of stimuli).

Dynamic properties. Stimuli were presented in trials that lasted 40 seconds. During this time, stimuli were updated dynamically with irregular refresh rates $(M=6.62 \mathrm{~Hz}, S D=$ 0.15). With no targets or TPDs present, distractors varied independently between the remaining 11 distractor colors and 5 distractor numbers. With every refresh, any stimulus could remain unchanged ( 0.6 probability), become more target-similar by one step $(0.2$ probability) or become less target-similar by one step ( 0.2 probability). The exception to this was when a distractor reached a point one step from a state reserved for targets or TPDs, and in such cases a distractor could only change to become less similar to a target. This resulted in a mean rate of change of $2.02 \mathrm{~Hz}(S D=0.54)$ for each distractor item.

The behavior of targets and TPDs was determined by a number of properties: (1) a time before a distractor began changing towards a target state; (2) a time before which a distractor became a target; and (3) a time for which a target remained present before reverting to a distractor if not detected. These timers were not defined as such but were implemented as randomly generated counters that were incrementally reduced until reaching zero (when the corresponding event would occur). In practice, the first color TPD became active after a mean of 6,566 ms $(S D=2,394)$, the first number TPD became active after a mean of 6,305 ms ( $S D$ $=2,440)$, the first color target onset after a mean of $18,169 \mathrm{~ms}(S D=6,453)$, the first number target onset after a mean of 16,222 ms $(S D=5,526)$, the second color target onset (where applicable) after a mean of 25,200 ms (SD = 5,018) and the second number target onset (where applicable) after a mean of 22,319 ms $(S D=4,496)$. The mean duration of color targets before a response was $7,649 \mathrm{~ms}(S D=3,099)$ and the mean duration of number targets 
before a response was $7,520 \mathrm{~ms}(S D=2,710)$. The specific parameters for the onset of targets were chosen so that no target would appear early after the beginning of a trial and not too late to allow monitoring.

\section{Design and Procedure}

A display configuration (contiguous-discrete) by search target (color-number-dual) within-participants design resulted in six blocked conditions. The side of the display on which the numbers appeared was counterbalanced between participants but remained constant for each participant. Participants were asked to search for a particular color target, a particular number target, or both a color and a number target. A target cue(s) was shown before every trial which was an example of that participant's target color, target number or both (dependent upon condition). In single-target (color only and number only) blocks there were six target absent trials, six trials with one target onset and six trials with two target onsets. In dual-target (color and number) blocks there were nine possible types of trial, one for each combination of zero, one or two color target onsets and zero, one or two number target onsets. Each of these nine trial types was shown twice. See Table 1 for a breakdown of the different target type and display configuration conditions and the number of each type of target possible within trials. Twelve minutes of eye movement data were generated for each block. See Figure 1 for details of trial procedure and responses.

\section{Results}

\section{Analyses and Exclusions}

To normalize distributions, all proportional data, including hit and false alarm rates, were arcsine-square-root transformed. Similarly, RT and visit duration data were log transformed. Untransformed means and standard deviations are reported. In the eye movement data, fixations were excluded from duration analyses if they were longer than 
1,200 ms or shorter than $80 \mathrm{~ms}$ in duration or if they corresponded with a manual response (6.15\% of all fixations).

\section{Behavioral Analyses}

The behavioral data were analyzed using 2 (target: single-target, dual-target) x 2 (stimulus configuration: contiguous, discrete) ANOVAs. Monitoring for both single-target color and number targets was compared against only responses to the matching target type from the dual-target conditions. Monitoring for color and number targets was analyzed separately in all cases. RT and false alarm rates are shown in Figure 2.

Hit rate. Hit rates were near ceiling for detecting color targets $(M=0.97, S D=0.04)$ and at ceiling for number targets, so no analyses of hit rates are presented.

Response time. There were significant main effects of target on RT for color, $F(1,25)$ $=17.57, p<.001, \eta^{2}{ }_{\mathrm{G}}=0.08$, and number targets, $F(1,25)=162.23, p<.001, \eta^{2}{ }_{\mathrm{G}}=0.50$. In both cases, responses were faster in single-target than dual-target conditions. The main effect of stimulus configuration failed to reach significance for responses to either color, $F(1,25)=$ 2.95, $p=.098$, or number targets, $F(1,25)=2.27, p=.145)$. The interaction between target and stimulus configuration approached significance for both color, $F(1,25)=0.30, p=.059$, $\eta^{2}{ }_{\mathrm{G}}=0.002$ and number targets, $F(1,25)=3.44, p=.075, \eta^{2}{ }_{\mathrm{G}}=0.03$. RTs to both color and number single-target condition were unaffected by display configuration. With respect to the dual-target conditions, there was a trend towards faster detection of color targets in contiguous than discrete displays and a trend towards faster detection of number targets in discrete than contiguous displays (see Figure 2).

False alarm rate. There were no significant main effects of target or stimulus configuration upon false alarm rate (all $F<1.92)$.

Behavioral summary. Monitoring for single-targets led to faster target detection than monitoring for dual-targets. The speed of target detection when monitoring for single-targets 
was unaffected by display configuration. In contrast, there were trends for color targets to be detected quickest when monitoring in the contiguous condition but for number targets to be detected quickest when monitoring in the discrete condition in the dual-target conditions.

\section{Eye Movement Analyses}

To examine predictive monitoring for potential targets, first fixations and refixations to targets and TPDs were analyzed, as were mean visit durations to the color and number sets (see Figures 3 and 4).

First fixations. The proportion of first fixations made to color and number targets and TPDs in each state were analyzed in two separate 3 (steps from target: $0,1,2$ ) x 2 (target: single-target, dual-target) x 2 (display configuration: contiguous, discrete) ANOVAs.

Color targets. The main effect of step, $F(2,50)=139.11, p<.001, \eta^{2}{ }_{\mathrm{G}}=0.68$, was significant, but the effects of target, $F(1,25)=2.07, p=.162$, and display configuration did not reach significance, $F(1,25)=0.04, p=.850$. The two-way interaction between target and step was significant, $F(2,50)=27.07, p<.001, \eta^{2} \mathrm{G}=0.12$, but the remaining interactions did not reach significance, $F<=1.81, p=>.174$. In the dual-target condition relative to the color only condition, a significantly greater proportion of color targets were first fixated when targets, $t(25)=6.62, p<.001$ and a significantly smaller proportion of forthcoming color targets were first fixated two steps from the target, $t(25)=5.12, p<.001$. There was no difference for those first fixated one step from the target, $t(25)=1.50, p=.147$.

Number targets. The main effect of step, $F(2,50)=124.74, p<.001, \eta^{2}{ }_{G}=0.46$, did reach significance, but the main effects of target, $F(1,25)=.89, p=.353$, and stimulus configuration did not, $F(1,25)=.09, p=.768$. The two-way interaction between target and step reached significance, $F(2,50)=5.56, p=.008, \eta^{2}{ }_{\mathrm{G}}=0.04$, as did the three-way interaction between step, task and display configuration, $F(2,50)=7.68, p=.001, \eta^{2}{ }_{G}=0.07$. 
No other interactions were significant, $F<=2.05, p>=.101$. A greater proportion of number targets were first fixated as targets when monitoring for numbers in the dual-target, relative to the single-target condition, $t(25)=3.33 p=.003$. When monitoring in discrete displays for both target types, relative to only numbers, a smaller proportion of number targets were first fixated one step from the target number, $t(25)=2.46, p=.021$, and two steps from the target number, $t(25)=3.30, p=.003$. However, when monitoring in contiguous displays, there was no difference between single- and dual-target conditions in the proportion of number targets first fixated one step from the target number, $t(25)=1.83, p=.079$, or two steps from the target number, $t(25)=0.36, p=.722$.

Refixations. The total number of fixations made to TPDs (summed across those one and two steps from a target state) were compared across single- and dual-target conditions for both color and number targets (see Figure 5). The data were analysed in 2 (task: single versus dual) x 2 (display configuration: contiguous versus discrete) ANOVAs repeated for color and number stimuli. For reference, color distractors (at least three steps from the target) received an average of $1.57(S D=0.18)$ fixations and number distractors (at least three steps from the target) received an average of $4.99(S D=1.28)$ fixations. In the case of the number set, this apparently high number of fixations may be due to the relatively small set size.

With respect to refixations to forthcoming color targets, the main effects of task, $F(1,25)=6.31, p=.019, \eta^{2}{ }_{\mathrm{G}}=0.02$, and display configuration, $F(1,25)=29.42, p<.001, \eta^{2}{ }_{\mathrm{G}}$ $=0.15$, were significant. However, the interaction between task and display configuration did not reach significance, $F(1,25)=1.84, p=.188$. Forthcoming color targets received fewer refixations in the dual-target $(M=4.75, S D=1.31)$ compared to single-target $(M=6.15, S D$ $=1.36)$ conditions and in the contiguous $(M=5.24, S D=1.72)$ compared to discrete $(M=$ $5.66, S D=1.56)$ conditions. 
With respect to refixations to forthcoming number targets, the main effect of display configuration was close to significance, $F(1,25)=3.98, p=.057$. However, neither the main effect of task, $F(1,25)=.69, p=.415$, nor the interaction between task and display configuration reached significance, $F(1,25)=1.84, p=.696$. Forthcoming number targets received marginally fewer refixations in discrete $(M=5.44, S D=3.44)$ compared to contiguous $(M=6.41, S D=3.29)$ configurations.

Visit durations. Visits were defined as groups of consecutive fixations made to a single stimulus set without fixating a location outside of that set (mean visit durations for each set in each condition are shown in Figure 3). When monitoring for a single target type, discrete displays led to longer mean visit durations to the relevant stimulus set for both color, $t(25)=23.04, \mathrm{p}<.001$, and number targets, $t(25)=2.76, p=.011$, relative to contiguous displays. Longer visits were made to the irrelevant number set when monitoring for only color targets in contiguous, relative to discrete, displays, $t(22)=7.71, p<.001$. There was no comparable effect when monitoring for only number targets, $t(18)=1.11, p<.281$. When monitoring in the dual-target conditions, mean visit durations to the number set were longer in contiguous than discrete displays, $t(25)=5.45, p<.001$. However, mean visit durations to the color set were longer in discrete compared to contiguous displays, $t(25)=5.27, p<.001$.

\section{Eye Movement Summary}

The response time data show that monitoring for color and number targets in dualtarget conditions was slowed relative to the single-target conditions. There was also a trend in dual-target conditions for responses to color targets to be speeded in contiguous than discrete displays, and for responses to number targets to be speeded in discrete than contiguous displays. These findings correspond with the eye movement data.

Visit durations in the single target conditions were longer in discrete relative to contiguous displays, and numbers were hard to ignore during color target search in 
contiguous displays. In dual-target search, contiguous displays increased visit durations to the number array but reduced them to the color array.

With respect to color targets, first fixations and refixations were both much less likely to be made to forthcoming targets in dual- than single-target search and refixations to forthcoming color targets were reduced in contiguous relative to discrete configurations. With respect to number targets, first fixations being made to forthcoming targets were reduced in the dual- relative to single-target search, but only in discrete displays. Discrete displays also reduced refixations to forthcoming number targets. Eye movements to number targets in contiguous displays were unaffected by target.

Considering the behavioral and eye movement results together, the dual-task conditions were associated with slowed detection of target onsets and reduced evidence of predictive fixations. Single-target searches in contiguous displays benefited from predictive monitoring but, in the case of color, involved difficulties maintaining focus on the correct stimulus set. With respect to dual-task conditions, the contiguous display configuration was associated with shorter visit durations to the color set but showed a trend towards speeded RTs to color targets and slowed RTs to number targets, relative to the discrete display configuration. Overall, dual-target searches slowed target detection, and predictive monitoring was much reduced in every dual-target condition other than when detecting numbers in contiguous displays.

\section{The Behavioural Impact of Predictive Monitoring}

The eye movement data show the most robust evidence of predictive monitoring in single-target conditions and these conditions were also associated with faster RTs (relative to dual-target conditions). Here we explore this association between predictive monitoring and behavioral performance in more detail, examining the effect of predicting forthcoming targets on RT and false alarm rate relative to responses to items first fixated as targets (these data are 
shown in Figure 6). As with the basic behavioral analysis hit rates were near ceiling and were not analyzed.

Color targets. There was no significant difference in RT between color targets first fixated as items one step from becoming targets and those first fixated as targets for: (1) single-target color search in contiguous displays, $t(25)=.07, p=.941$; (2) single-target color search in discrete displays, $t(25)=.94, p=.354$; (3) dual-target search in contiguous displays, $t(24)=1.19, p=.245$; and (4) dual-target search in discrete displays, $t(25)=.32, p=.753$. The false alarm rate for stimuli first fixated one step from becoming targets was significantly greater than zero for: (1) single-target color search in discrete displays, $t(25)=2.70, p=.012$; (2) dual-target search in contiguous displays, $t(25)=2.39, p=.025$; and (3) dual-target search in discrete displays, $t(25)=2.56, p=.017$. However, the false alarm rate for color stimuli first fixated as items one step from becoming targets was not significantly greater than zero for single-target color search in contiguous displays, $t(25)=1.57, p=.128$.

Number targets. There was no significant difference in RT between number targets first fixated as items one step from becoming targets and those first fixated as targets for: (1) single-target number search in contiguous displays, $t(20)=.44, p=.663$; (2) single-target number search in discrete displays, $t(18)=1.53, p=.143$; (3) dual-target search in contiguous displays, $t(21)=1.49, p=.152$; and (4) dual-target search in discrete displays, $t(19)=.33, p$ $=.747$. The false alarm rate for number stimuli first fixated as items one step from becoming targets was significantly greater than zero for: (1) single-target number search in contiguous displays, $t(25)=3.00, p=.006$; (2) single-target number search in discrete displays, $t(25)=$ 2.37, $p=.026$; (3) dual-target search in contiguous displays, $t(24)=2.79, p=.010$; and (4) dual-target search in discrete displays, $t(25)=2.94, p=.007$.

Summary. Evidence from eye movements showed predictive monitoring was more striking in the single-target conditions than the dual-target conditions and occurred alongside 
evidence of speeded detection of target onsets. However, a more detailed examination showed that predictive monitoring of specific targets did not speed RTs but was associated with false alarms across all conditions other than when monitoring for color targets in contiguous displays.

\section{Discussion}

The present study examined the cost of monitoring for the onset of targets from color and number categories relative to single-target baselines. It was hypothesized that target detection would be less accurate and eye movements associated with predictive monitoring would be reduced when searching for targets in two categories compared to one. How display configuration might influence this cost to accuracy and predictive monitoring in dual-target versus single-target search was also explored.

The results were broadly consistent with the hypotheses outlined in the Introduction. First, detection of target onsets was faster when monitoring for a single target of either type compared to both. Second, first fixations and refixations to forthcoming targets, i.e. eye movements which reflected predictive monitoring, were markedly reduced in dual-target search relative to single-target search. Third, in the dual-task condition, monitoring for color and number targets were differentially influenced by display configuration. Fourth, at least with respect to the monitoring for color targets, evidence of predictive monitoring was found for both discrete and contiguous displays. Fifth, evidence of predictive monitoring did not speed RTs to target onsets but did involve a risk of making false alarms. We now consider each of these conclusions in turn.

The slowed RTs in dual-, relative to single-target, conditions are consistent with previous findings. While responses were slowed relative to monitoring for a single target type, it is important to note that other indices of performance such as hit and false alarm rates 
were unaffected by the introduction of an additional target category. With respect to the present study, we consider the cost to reflect maintaining or switching between two task sets, where each task set includes specific target templates. Relative to single-target baselines, monitoring for an additional target category requires defining an additional template, and storing or accessing color and number templates with working memory (WM). In support of this explanation is a body of evidence on the costs to attentional guidance when having to search for more than one color target simultaneously (Godwin, Menneer, Cave, \& Donnelly, 2010; Menneer et al., 2008; Stroud et al., 2012, 2011). In the present case, monitoring for both color and number target onsets must rely on holding two sets of target representations and alternating between them.

Evidence of predictive monitoring for targets was found to be reduced in the dualtarget conditions. Elsewhere we have suggested that predictive monitoring requires the specification of broad and specific target templates (Muhl-Richardson et al., in press). Within a category, a broad template includes a range of colors (or numbers) and identifies items that might be targets. Once potential targets are identified, a second, more specific, template is used to evaluate target identity. The notion of broad and specific target templates places a load on WM. Here we suggest that either the memory load is too great, or the coordination too difficult, to use both broad and specific templates effectively when monitoring for two categories of target. In either case, the specific nature of the demands placed on WM by such a set of target templates warrants further investigation.

Display configuration markedly influenced eye movement strategies used in monitoring for target onsets. In all single-target conditions, discrete displays supported longer visit durations to the relevant stimulus set than in contiguous displays. This finding is unsurprising and consistent with the expectation that participants would rarely attend to a second, more spatially distant, and irrelevant stimulus set in single-target searches. More 
specifically, in the single-target color conditions, contiguous displays also led to reduced refixations of forthcoming targets relative to discrete displays. In contrast, in the single-target number conditions, refixations to forthcoming targets were reduced in discrete relative to contiguous displays. In sum, discrete configurations supported focused monitoring in the single-target conditions. The advantage to single-target monitoring with discrete configurations reflects the capacity to focus attention. In the case of contiguous configurations, the central position of the number items and the more peripheral position of the color items either allowed fixations to drift to the number or for an effort to be made to monitor for color targets in peripheral vision. In either case, contiguous configurations presented a challenge to the focusing of attention.

In dual-target conditions, there was a trend towards faster RTs to color targets in contiguous displays and to number targets in discrete displays (in both cases relative to the other display configuration). These trends occurred despite fixations being most focused on the number set in contiguous configurations of the dual-target condition. This same condition was also associated with reduced evidence of predictive monitoring for the onset of number targets, which likely results from the effort to monitor for color target onsets using a broadened attentional field in contiguous configurations. The fact that display configuration influenced monitoring strategy for targets onsets is a highly important consideration in realworld monitoring tasks. The specific influence of display configuration, especially in dualtarget scenarios, warrants investigation with more comprehensive set of display manipulations and we do not attempt to draw such conclusions in the present case. Furthermore, in the real-world, monitoring processes are very likely to be subject to influence from interactions with fatigue and stress. Although the present data do not allow exploration of these issues, we note their potential importance for future work. 
While one might think that predictive monitoring should lead to speeded RTs relative to those items first fixated as targets, no evidence of such an effect was found. RTs were the same to targets first fixated pre-onset relative to those first fixated as targets. The lack of an apparent behavioral benefit to predictive monitoring on this measure is further highlighted by the fact that those items first fixated one step from being targets were subject to a measurable false alarm rate, which may be taken to reflect a tendency to make overly fast decisions when monitoring for targets. We suggest, however, that this apparent paradox is less striking than it might seem at first glance. The present study involved variable target onsets allied with a low overall level of target prevalence, especially when considered in terms of the proportion of total trial time for which targets were present, rather than the proportion of target-present trials. This meant that participants would have been foolhardy to adopt a wait-and-see strategy once locating a potential target, as the reliability of TPDs as predictive cues was variable. Rather, they should have, and did, identify potential targets and monitor them, returning to them with multiple refixations over time. Such a strategy inevitably involves the risk that targets may not have been fixated at the time of onset.

While a low level of target prevalence may have contributed to the lack of an observable behavioral benefit to predictive monitoring in the present study, it is the case that there is a spectrum of target prevalence across real-world monitoring tasks. In some tasks, such as monitoring military radar, targets may be rare and it may be difficult to observe a behavioral benefit to predictive monitoring. In other tasks, such as the geological screening outlined in the Introduction, targets may be more common and it is likely that a behavioral benefit to predictive monitoring would be observable and that this would be subject to the influence of display configuration similar to that observed here in terms of eye movements. In other words, the extent to which predictive monitoring might afford a behavioral benefit in 
the real-world is likely to depend primarily upon the prevalence parameters of the particular task, but also, in dual-target scenarios, upon the specific spatial configuration.

In conclusion, the present study provides a first insight into how predictive monitoring is managed in the case of search for two target categories. These findings have implications, across a range of applied domains, for the design of electronic visual displays that integrate multiple categories of information. If a secondary source of information can be closely integrated with a primary source, in a way that minimizes attentional shifts, then this may facilitate the prediction of target information. In the example of marine radar, this might involve the integration or overlaying of alphanumeric indicators of position, direction and speed within a central map, rather than positioning these values peripherally within discrete regions. Furthermore, with recent advances in the availability and popularity of augmented and virtual reality devices, this is not just a problem faced by those in highly specific applied scenarios, but by user interface designers more broadly. In taking advantage of the flexibility offered by modern computing and display technology, it is important for developers and designers to determine the optimal way to integrate different sources of complex visual information. Regardless of the specific application, it is difficult to imagine a case where facilitating effective attentional guidance would not be desirable. 


\section{References}

Bach, M. (1996). The Freiburg Visual Acuity test--automatic measurement of visual acuity. Optometry and Vision Science, 73(1), 49-53.

Donnelly, N., Cave, K. R., Welland, M., \& Menneer, T. (2006). Breast screening, chicken sexing and the search for oil: challenges for visual cognition. Geological Society, London, Special Publications, 254(1), 43-55. http://doi.org/10.1144/gsl.sp.2006.254.01.04

Fletcher, R. (1998). The City University Colour Vision Test (3rd ed.). London: Keeler.

Godwin, H. J., Hout, M. C., \& Menneer, T. (2014). Visual similarity is stronger than semantic similarity in guiding visual search for numbers. Psychonomic Bulletin \& Review, 21(3), 689-695. http://doi.org/10.3758/s13423-013-0547-4

Godwin, H. J., Menneer, T., Cave, K. R., \& Donnelly, N. (2010). Dual-target search for high and low prevalence X-ray threat targets. Visual Cognition, 18(10), 1439-1463. http://doi.org/10.1080/13506285.2010.500605

Kiesel, A., Steinhauser, M., Wendt, M., Falkenstein, M., Jost, K., Philipp, A. M., \& Koch, I. (2010). Control and interference in task switching-A review. Psychological Bulletin, 136(5), 849-874. http://doi.org/10.1037/a0019842

Lavie, N. (2006). The role of perceptual load in visual awareness. Brain Research, 1080(1), 91-100. http://doi.org/10.1016/j.brainres.2005.10.023

Lavie, N., Beck, D. M., \& Konstantinou, N. (2014). Blinded by the load : attention, awareness and the role of perceptual load Blinded by the load : attention, awareness and the role of perceptual load. Philos Trans R Soc Lond B Biol Sci, 369(1641), 20130205. 
http://doi.org/10.1098/rstb.2013.0205

Meiran, N. (2000). Modeling cognitive control in task-switching. Psychological Research, 63(3-4), 234-249. http://doi.org/10.1007/s004269900004

Meiran, N., Chorev, Z., \& Sapir, A. (2000). Component Processes in Task Switching. Cognitive Psychology, 41(3), 211-253. http://doi.org/10.1006/cogp.2000.0736

Menneer, T., Phillips, L., Donnelly, N., Barrett, D. J. K., \& Cave, K. R. (2004). Search efficiency for multiple targets. Cognitive Technology, 9(2), 22-25.

Menneer, T., Stroud, M. J., Cave, K. R., Donnelly, N., \& Rayner, K. (2008). Eye movements in search for multiple targets. In K. Rayner, D. Shen, X. Bai, \& G. Yan (Eds.), Cognitive and Cultural Influences on Eye Movements. London: Psychology Press.

Muhl-Richardson, A., Godwin, H. J., Garner, M., Hadwin, J. A., Liversedge, S. P., \& Donnelly, N. (n.d.). Individual Differences in Search and Monitoring for Color Targets in Dynamic Visual Displays. Journal of Experimental Psychology: Applied.

Pashler, H. (2000). Task switching and multitask performance. In S. Monsell \& J. Driver (Eds.), Attention and Performance XVIII: Control of Cognitive Processes (pp. 277-307). Cambridge, MA: MIT Press.

Ravizza, S. M., \& Carter, C. S. (2008). Shifting set about task switching: Behavioral and neural evidence for distinct forms of cognitive flexibility. Neuropsychologia, 46(12), 2924-2935. http://doi.org/10.1016/j.neuropsychologia.2008.06.006

Sobel, K. V., Puri, A. M., \& Hogan, J. (2014). Target grouping in visual search for multiple digits. Attention, Perception, \& Psychophysics, 77(1), 67-77. http://doi.org/10.3758/s13414-014-0761-9

Stroud, M. J., Menneer, T., Cave, K. R., \& Donnelly, N. (2012). Using the dual-target cost to 
explore the nature of search target representations. Journal of Experimental Psychology: Human Perception and Performance, 38(1), 113-122. http://doi.org/10.1037/a0025887

Stroud, M. J., Menneer, T., Cave, K. R., Donnelly, N., \& Rayner, K. (2011). Search for multiple targets of different colours: misguided eye movements reveal a reduction of colour selectivity. Applied Cognitive Psychology, 25(6), 971-982. 
Table 1. The different combinations of target type and display configuration (manipulated between blocks) and the number of each target type that could appear per trial (counterbalanced within blocks).

\begin{tabular}{l|lll} 
Target & Color & Number & Color and number \\
$\begin{array}{l}\text { Display } \\
\text { Configuration }\end{array}$ & $\begin{array}{l}\text { Contiguous or } \\
\text { discrete }\end{array}$ & $\begin{array}{l}\text { Contiguous or } \\
\text { discrete }\end{array}$ & $\begin{array}{l}\text { Contiguous or } \\
\text { discrete }\end{array}$ \\
$\begin{array}{l}\text { Color Targets } \\
\text { Present Per Trial }\end{array}$ & 0,1 or 2 & 0 & 0,1 or 2 \\
$\begin{array}{l}\text { Number Targets } \\
\text { Present Per Trial }\end{array}$ & 0 & 0,1 or 2 & 0,1 or 2
\end{tabular}


a)

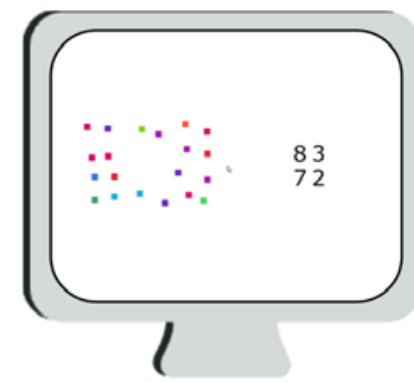

b)

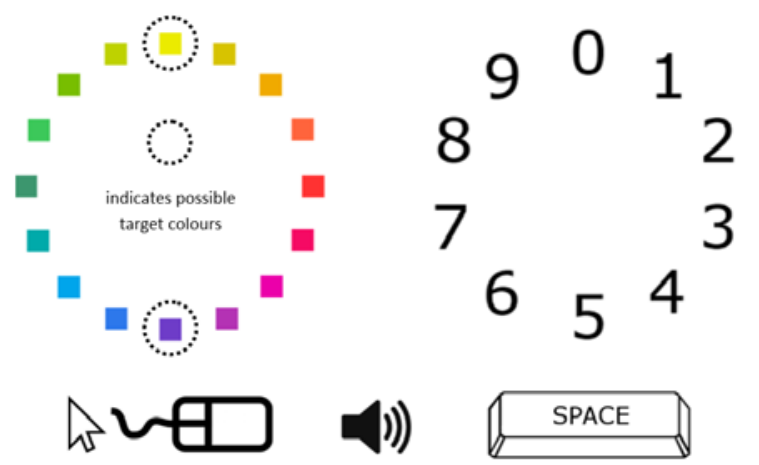

c)

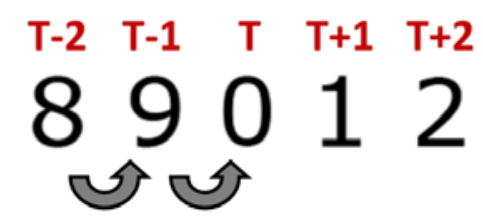

d)

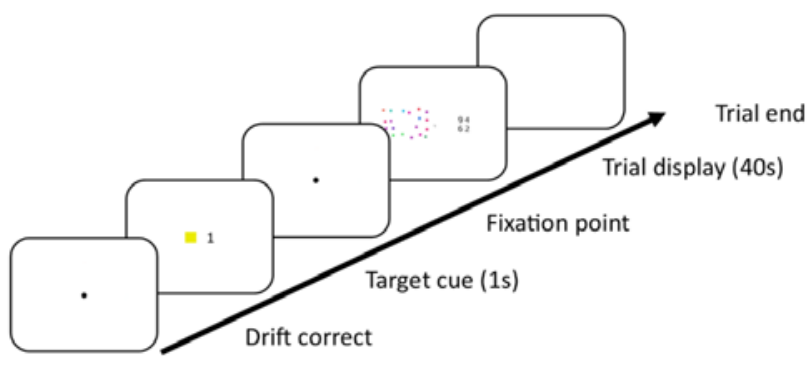

a) Displays consisted of colored squares and black digits in discrete (left) and contiguous (right) configurations.

b) Color stimuli were defined on a 16 -item scale (Menneer et al., 2007; 2010, Stroud et al., 2011; 2012).

Number stimuli were defined on a scale of 0 to 9 in the font Veranda (Godwin, Hout \& Menneer, 2014). Color targets could be one of two opposing colors: yellow ( $x=0.400$, $y=0.496)$ or purple $(x=0.207$, $y=0.128)$. Number targets could be any digit. Color responses were made by mouse-clicks directly on targets and number responses were a spacebar press (different responses were used to minimize response-based interference). All responses generated audio feedback.

c) Each participant was assigned consistent targets (examples shown). Stimuli only changed by single steps, so the stimuli one or two steps from the target were potentially target-predictive.

d) Each trial was preceded by a target cue, followed by a central fixation point. Trials lasted for 40 seconds and there were 18 trials in each of the six blocks. Three practice trials were given before each block and trial order was randomized.

Figure 1. Method infographic 
RT (colour targets)

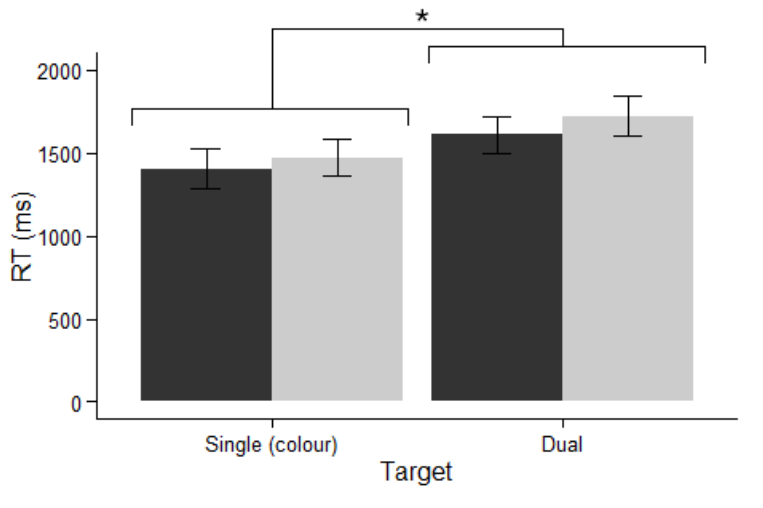

Display Configuration
RT (number targets)

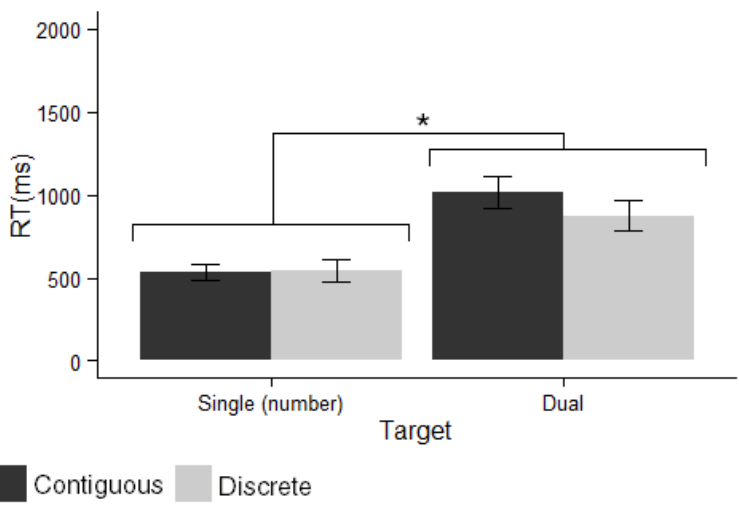

False Alarm Rate (number targets)

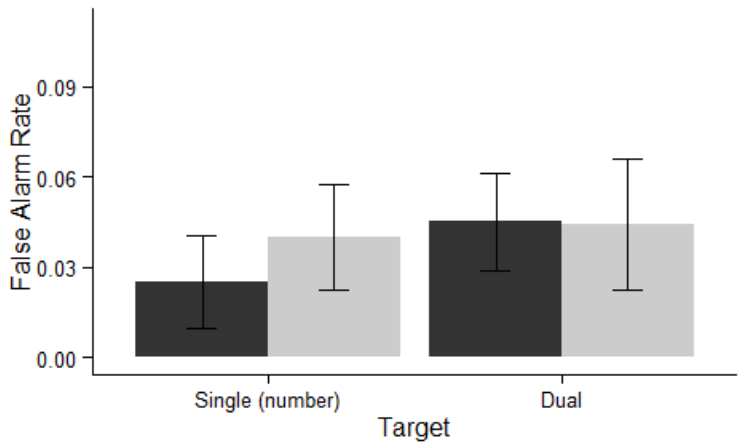

Figure 2. RTs and false alarm rates for all target types in all display configurations (error bars show 95\% CIs and ' $*$ ' indicates a pairwise comparison where $p<.05$ ). 


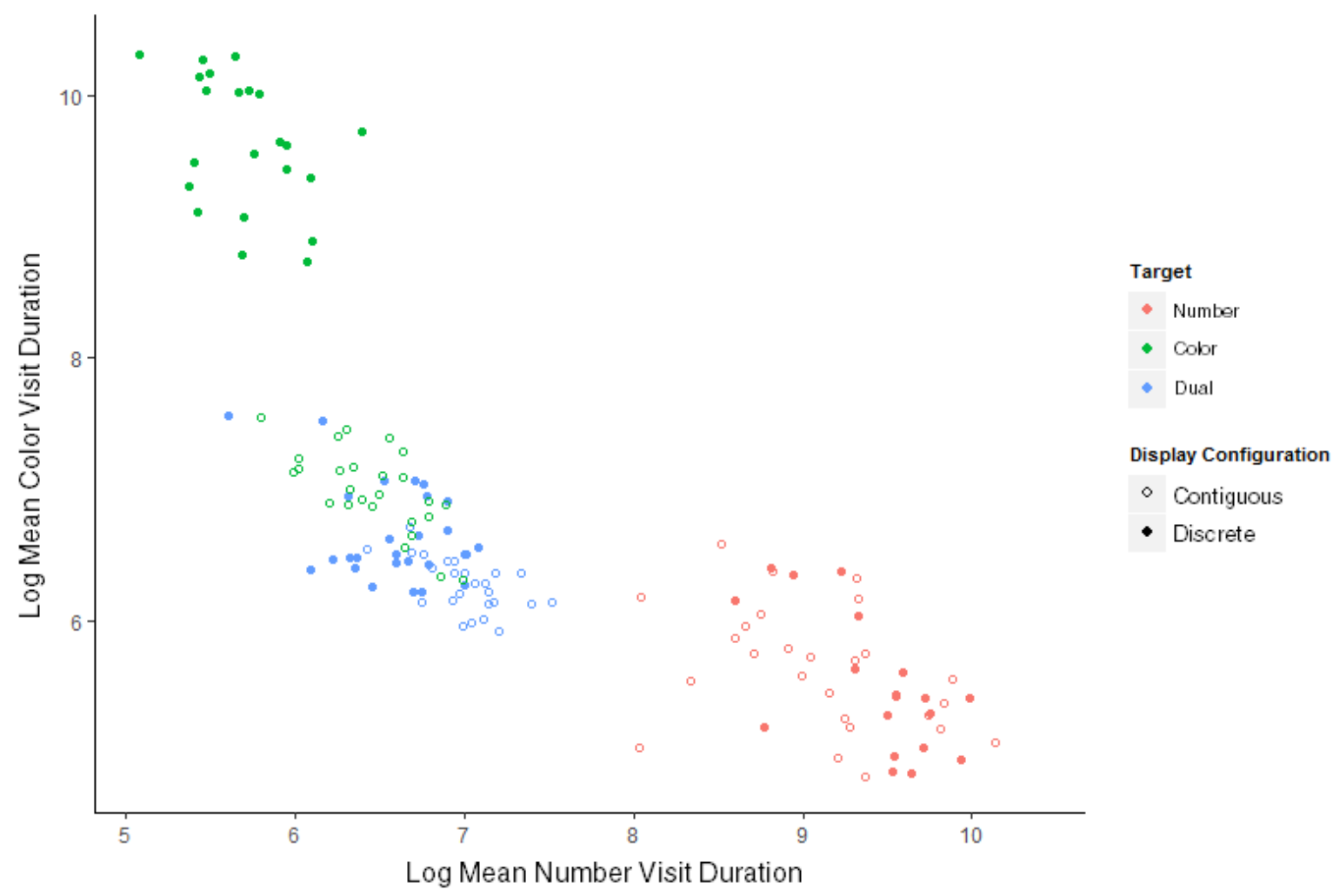

Figure 3. Log mean visit durations to color (y-axis) and number (x-axis) stimulus arrays in number only, color only and dual-target search across both contiguous and discrete displays. 

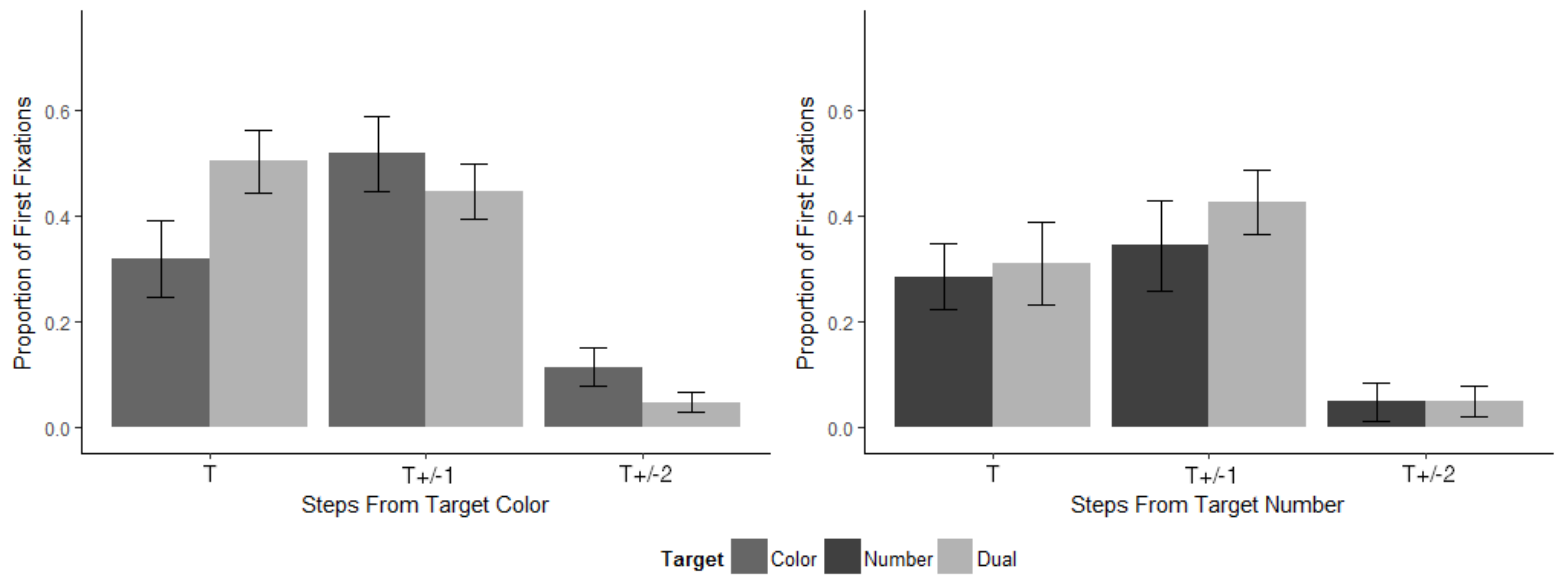

Colour Target First Fixations (discrete displays) Number Target First Fixations (discrete displays)
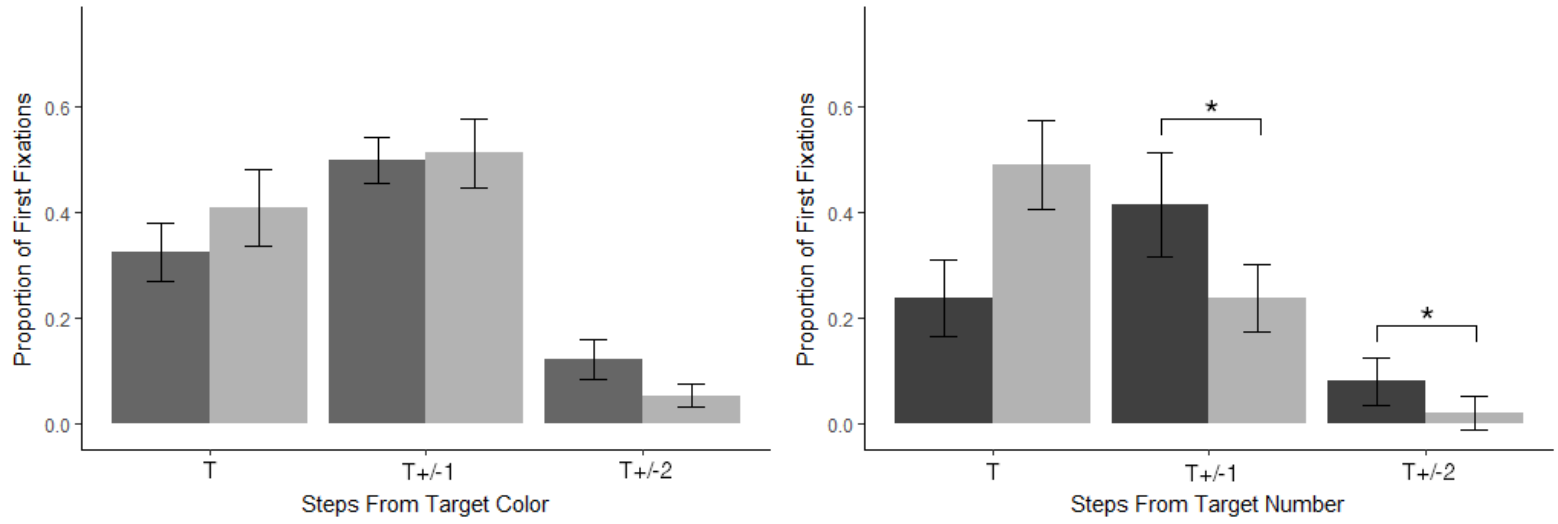

Figure 4. Proportion of first fixations to targets by step from the target in all search and display types (error bars show 95\% CIs and '*' indicates a pairwise comparison where $\mathrm{p}<$ .05; additional significant pairwise comparisons that were not carried out at each level of display configuration are not shown but are reported in the text). 


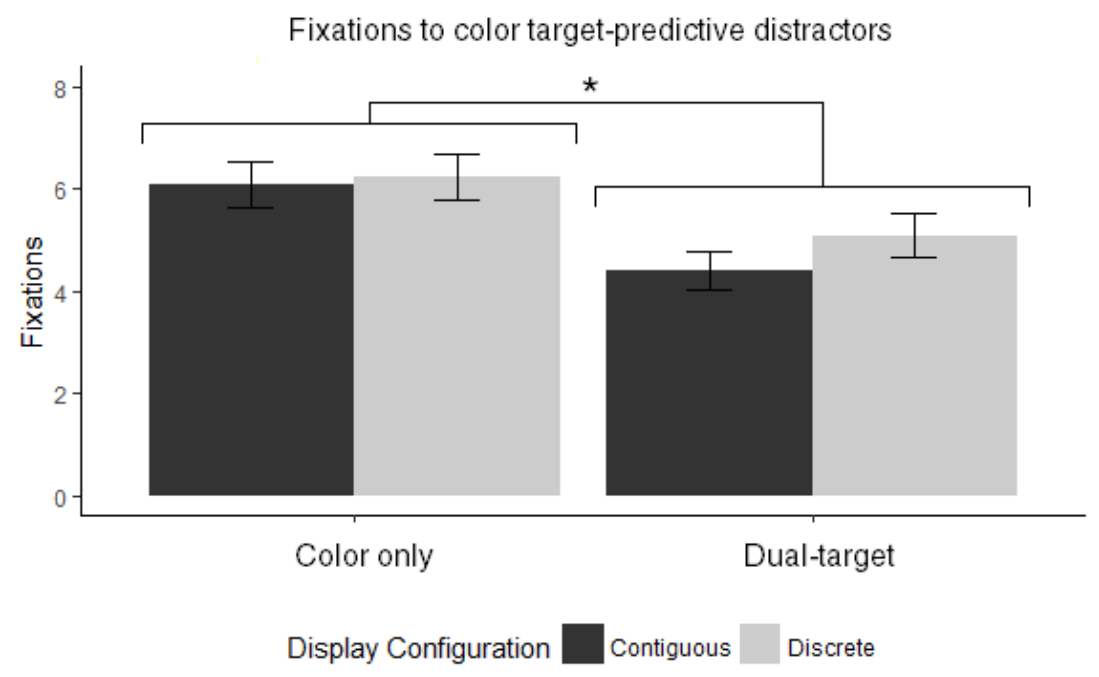

Fixations to number target-predictive distractors

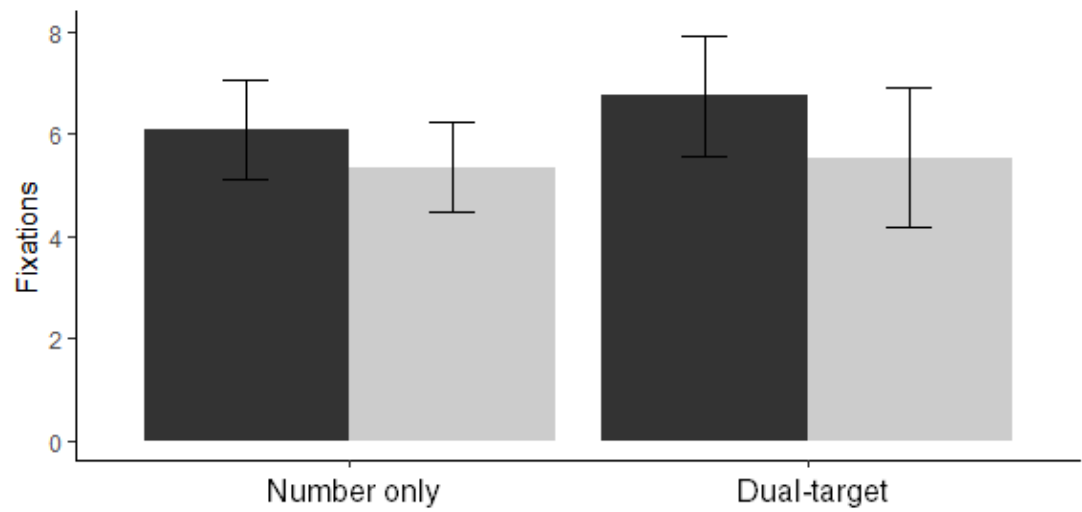

Figure 5. Total number of fixations to forthcoming color (top) and number (number) targets summed between those first fixated as TPDs at $\mathrm{T}+/-1$ and $\mathrm{T}+/-2$ steps from a target state (error bars show 95\% CIs). 

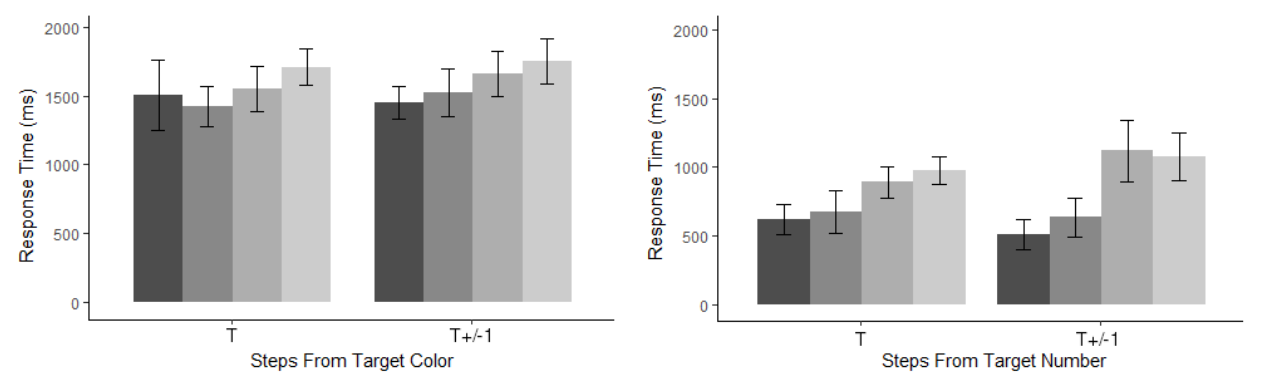

Display Configuration/Target

teps From Target Color
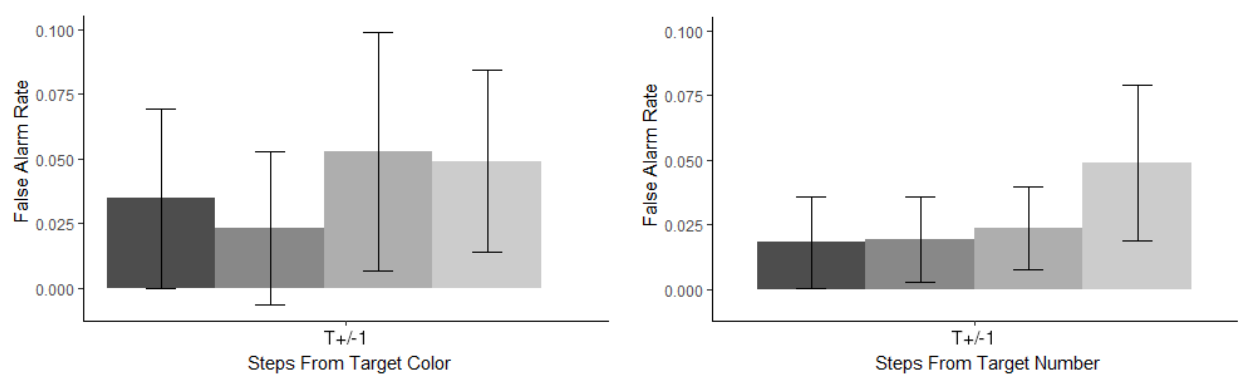

Single Target/Contiguou

Single Target/Discrete

Dual Target/Contiguous

Dual Target/Discrete

Figure 6. Response time (RT) for forthcoming color and number targets first fixated at $\mathrm{T}$ and as TPDs at T+/-1 step (top) and false alarm rate for TPDs first fixated at $\mathrm{T}+/-1$ (bottom; error bars show 95\% CIs). 